7. Papamatheakis DG, Mocumbi AOH, Kim NH, Mandel J. Schistosomiasis-associated pulmonary hypertension. Pulm Circ. 2014;4:596-611. http://dx.doi.org/10.1086/678507

8. Gobbi F, Buonfrate D, Angheben A, Beltrame A, Bassetti M, Bertolaccini L, et al. Pulmonary nodules in African migrants caused by chronic schistosomiasis. Lancet Infect Dis. 2017;17:e159-65. http://dx.doi.org/10.1016/S1473-3099(16)30530-8

9. Bethlem EP, Schettino GP, Carvalho CR. Pulmonary schistosomiasis. Curr Opin Pulm Med. 1997;3:361-5. http://dx.doi.org/10.1097/ 00063198-199709000-00008

10. Gobbi F, Formenti F, Perandin F, Buonfrate D, Angheben A, Paiano $\mathrm{S}$, et al. Real-time polymerase chain reaction assay on bronchoalveolar lavage: an alternative method for diagnosing chronic pulmonary schistosomiasis? Am J Trop Med Hyg. 2017;97:1808-9. http://dx.doi.org/10.4269/ajtmh.17-0499

Address for correspondence: Anastasia Saade, Hopital Cochin, 27 Rue du Faubourg Saint-Jacques, 75014 Paris, Île-de-France, France; email: anastasia.saade@gmail.com

\section{Strongyloidiasis and Culture-Negative Suppurative Meningitis, Japan, 1993-2015}

\author{
Mitsuru Mukaigawara, Izumi Nakayama, \\ Koichiro Gibo
}

Author affiliation: Okinawa Chubu Hospital, Okinawa, Japan

DOI: https://doi.org/10.3201/eid2412.180375

Community-acquired Enterobacteriaceae infection and culture-negative meningitis are rare and atypical subtypes of meningitis in adults. Of 37 patients who had atypical suppurative meningitis during 1993-2015 in Okinawa, Japan, $54.5 \%$ had strongyloidiasis, of which $9.1 \%$ cases were hyperinfections and $3.0 \%$ dissemination. Strongyloidiasis should be considered an underlying cause of atypical suppurative meningitis.

A mong adults, suppurative meningitis caused by enteric organisms and suppurative meningitis that is culture negative are uncommon $(1,2)$. These types of meningitis with atypical features (hereafter atypical suppurative meningitis) remain a clinical challenge. The mortality rate among patients with community-acquired suppurative meningitis caused by gram-negative organisms is $52.5 \%$ (1). Treatment of culture-negative suppurative meningitis requires broad-spectrum antimicrobial drugs; however, the absence of detected pathogens increases the risk for development of antimicrobial drug resistance.

Strongyloidiasis, a nematode infection that occurs in the subtropics and tropics, is associated with Enterobacteriaceae meningitis (3). Previous reports of strongyloidiasis-associated meningitis also suggested potential links between strongyloidiasis and atypical suppurative meningitis on the basis of 9 cases of Enterobacteriaceae meningitis (not consecutive) (4) and 17 cases of culture-negative suppurative meningitis (5). Our aim was to investigate the association between strongyloidiasis and atypical suppurative meningitis.

We conducted a retrospective chart review of patients who consecutively received a diagnosis of atypical suppurative meningitis during January 1993-December 2015 at Okinawa Chubu Hospital, Okinawa, Japan. This hospital is one of the largest tertiary medical centers in Okinawa. Strongyloidiasis is endemic in Okinawa; reported prevalence is $5.2 \%(6)$.

We defined atypical suppurative meningitis as suppurative meningitis with positive cerebrospinal fluid (CSF) culture results for enteric organisms or with CSF leukocytosis $\geq 500$ cells $/ \mathrm{mm}^{3}$ and negative CSF culture results. We included in the study patients $\geq 18$ years of age with CSF that was culture positive for enteric organisms or negative with leukocytosis of $\geq 500 \mathrm{cells} / \mathrm{mm}^{3}$. Enteric organisms included in this study were Bacteroides spp., Enterococcus spp., Escherichia coli, Enterobacter spp., Klebsiella spp., Bifidobacterium bifidum, Clostridium perfringens, Proteus mirabilis, Streptococcus gallolyticus (bovis), and Campylobacter spp. (7). CSF of patients with bacterial meningitis typically shows leukocytosis of $\geq 1,000$ cells/ $\mathrm{mm}^{3}$; CSF of those with nonbacterial meningitis typically shows $<250$ cells $/ \mathrm{mm}^{3}(8)$. Considering the early phase of bacterial meningitis (9), the cutoff value (500 cells/ $\mathrm{mm}^{3}$ ) was defined to include suppurative meningitis and exclude most cases of nonbacterial meningitis. We excluded patients with nosocomial meningitis, prior use of antimicrobial drugs (within 7 days of lumbar puncture), negative CSF culture, and positive blood culture for nonenteric organisms.

We collected information about patient demographic and clinical characteristics, immunocompromised status, type of strongyloidiasis infection, outcomes, CSF analysis results, and culture results. Strongyloidiasis was classified into 3 categories: nonsystemic strongyloidiasis, hyperinfection, and dissemination. We defined these categories according to where larvae were detected: nonsystemic strongyloidiasis in fecal samples only, hyperinfection in sputum, and dissemination in samples other than feces or sputum (4). Samples were collected with regard to patients' clinical category. Identifying information was removed before 
analysis. This study was approved by the Okinawa Chubu Hospital Institutional Review Board (H29-76).

We identified 37 patients; CSF culture results were positive for Enterobacteriaceae for 14 and negative for 23. Strongyloidiasis was diagnosed by parasitologic examinations (Table; online Technical Appendix, https://wwwnc. cdc.gov/EID/article/24/12/18-0375-Techapp1.pdf).

Patients were 19-91 years of age (median 60 years of age); $59.5 \%$ (22/37) were male. Common chief complaints included headache (64.9\% [24/37]) and fever (59.5\% [22/37]). Human T-cell lymphotropic virus type 1 serologic test results were available for 31 patients, of which $22(71.0 \%)$ were positive. Of the 37 total patients, $3(8.1 \%)$ patients had diabetes mellitus, $3(8.1 \%)$ had cirrhosis, and $4(10.8 \%)$ used steroids on a regular basis. Parasitologic examinations were performed for 33 patients, and strongyloides were found in $18(54.5 \%): 14(42.4 \%)$ nonsystemic strongyloidiasis, 3 (9.1\%) hyperinfection, and $1(3.0 \%)$ dissemination.

Among 14 culture-positive patients, 5 (35.7\%) had nonsystemic strongyloidiasis and $3(21.4 \%)$ had hyperinfection. Among 19 culture-negative patients, 9 (47.4\%) had nonsystemic strongyloidiasis and 1 (5.3\%) had dissemination. When culture-positive patients with strongyloidiasis were compared with culture-negative patients with strongyloidiasis, the odds ratio was 1.71 (95\% CI $0.37-8.22)$. Of note, all strongyloidiasis-positive patients were born before 1960, suggesting changes in lifestyle and the environment since then (e.g., reduced exposure to contaminated soil during farming by not walking barefoot and improved farming environments).

\begin{tabular}{|c|c|c|c|}
\hline Characteristic & $\begin{array}{l}\text { All cases, no. }(\%) \\
n=37\end{array}$ & $\begin{array}{l}\text { Culture-positive, no. (\%), } \\
n=14\end{array}$ & $\begin{array}{l}\text { Culture-negative, no. (\%), } \\
n=23\end{array}$ \\
\hline \multicolumn{4}{|l|}{ Demographic and clinical } \\
\hline \multicolumn{4}{|l|}{ Sex } \\
\hline M & $22(59.5)$ & $9(64.2)$ & $13(56.5)$ \\
\hline $\mathrm{F}$ & $15(40.5)$ & $5(35.8)$ & $10(43.5)$ \\
\hline \multicolumn{4}{|l|}{ Chief complaints } \\
\hline Headache & $24(64.9)$ & $6(42.9)$ & $18(78.3)$ \\
\hline Fever & $22(59.5)$ & $9(64.2)$ & $13(56.5)$ \\
\hline Altered mental status & $8(21.6)$ & $5(35.7)$ & $3(13.0)$ \\
\hline Nausea/vomiting & $8(21.6)$ & $1(7.1)$ & $7(30.4)$ \\
\hline \multicolumn{4}{|l|}{ Immunocompromised status } \\
\hline HTLV-1 infectiont & $22(71.0)$ & $8(61.5)$ & $14(77.8)$ \\
\hline Diabetes mellitus & $3(8.1)$ & $2(14.3)$ & $1(4.3)$ \\
\hline Cirrhosis & $3(8.1)$ & $3(21.4)$ & 0 \\
\hline Steroid use & $4(10.8)$ & $1(7.1)$ & $3(13.0)$ \\
\hline \multicolumn{4}{|l|}{ Strongyloidiasis infection type } \\
\hline Nonsystemic strongyloidiasis & $14(42.4)$ & $5(35.7)$ & $9(47.4)$ \\
\hline Hyperinfection & $3(9.1)$ & $3(21.4)$ & 0 \\
\hline Dissemination & $1(3.0)$ & 0 & $1(5.3)$ \\
\hline Not analyzed & 4 & 0 & 4 \\
\hline Died & $5(13.5)$ & $3(21.4)$ & $2(8.7)$ \\
\hline \multicolumn{4}{|l|}{ CSF } \\
\hline \multicolumn{4}{|l|}{ Neutrophils $/ \mathrm{mm}^{3}$} \\
\hline $500-2,999$ & $24(64.9)$ & $6(42.9)$ & $18(78.3)$ \\
\hline $3,000-5,999$ & $4(10.8)$ & $1(7.1)$ & $3(13.0)$ \\
\hline $6,000-9,999$ & $2(5.4)$ & $1(7.1)$ & $1(4.3)$ \\
\hline$>10,000$ & $3(8.1)$ & $2(14.3)$ & $1(4.3)$ \\
\hline Glücose $<40 \mathrm{mg} / \mathrm{dL}$ & $14(37.8)$ & $7(50.0)$ & $7(30.4)$ \\
\hline \multicolumn{4}{|l|}{ Bacteriologic } \\
\hline \multicolumn{4}{|l|}{ Blood culture } \\
\hline Klebsiella pneumoniae & $7(18.9)$ & $5(35.7)$ & $2(8.7)$ \\
\hline Streptococcus gallolyticus & $4(10.8)$ & $4(28.6)$ & 0 \\
\hline Escherichia coli & $3(8.1)$ & $2(14.3)$ & $1(4.3)$ \\
\hline Streptococcus infantarius & $1(2.7)$ & $1(7.1)$ & 0 \\
\hline Campylobacter fetus & $1(2.7)$ & $1(7.1)$ & 0 \\
\hline Negative & $21(56.8)$ & $1(7.1)$ & $20(87.0)$ \\
\hline \multicolumn{4}{|l|}{ CSF culture } \\
\hline K. pneumoniae & $5(35.7)$ & $5(35.7)$ & 0 \\
\hline S. gallolyticus & $1(7.1)$ & $1(7.1)$ & 0 \\
\hline E. coli & $1(7.1)$ & $1(7.1)$ & 0 \\
\hline S. infantarius & $1(7.1)$ & $1(7.1)$ & 0 \\
\hline C. fetus & $1(7.1)$ & $1(7.1)$ & 0 \\
\hline Bacteroides fragilis & $1(7.1)$ & $1(7.1)$ & 0 \\
\hline
\end{tabular}

${ }^{*}$ Patient median age (range): all, 60 y (19-91 y); culture-positive, 57 y (27-91 y); culture-negative, 58 y (19-90 y). CSF, cerebrospinal fluid; HTLV-1, human T-cell lymphotropic virus type 1.

†HTLV-1 serology results were available for $93 \%$ (13/14) of culture-positive patients and $78 \%(18 / 23)$ of culture-negative patients. 
In patients with culture-positive meningitis, blood and CSF culture results were positive for Klebsiella pneumoniae, S. gallolyticus, E. coli, Streptococcus infantarius, and Campylobacter fetus. Among patients with culture-negative meningitis, blood culture results were positive for $K$. pneumoniae and E. coli.

Our investigation has several limitations because of the single-center, retrospective nature of this study. Also, the sensitivity of parasitologic examination is low (10). We potentially underestimated the prevalence of strongyloidiasis.

On the basis of previous reports of associations between strongyloidiasis and Enterobacteriaceae meningitis $(3,5)$, our analysis proposes an association between strongyloidiasis and culture-negative suppurative meningitis in Okinawa. Our findings suggest that atypical suppurative meningitis can occur as occult dissemination $(3,4)$, that anthelminthic treatment may be indicated, and that steroids should be administered with caution (5). The presence of atypical suppurative meningitis in adults should prompt consideration of occult disseminated strongyloidiasis; the index of suspicion for patients with atypical suppurative meningitis is high.

\section{About the Author \\ Dr. Mukaigawara is a chief medical resident at Okinawa Chubu Hospital. His primary research interests include the roles of international organizations in reducing global health inequities and clinical epidemiology of diseases in the Pacific region, including strongyloidiasis.}

\section{References}

1. Pomar V, Benito N, López-Contreras J, Coll P, Gurguí M, Domingo P. Spontaneous gram-negative bacillary meningitis in adult patients: characteristics and outcome. BMC Infect Dis. 2013;13:451. http://dx.doi.org/10.1186/1471-2334-13-451

2. Sigurdardóttir B, Björnsson OM, Jónsdóttir KE, Erlendsdóttir H, Gudmundsson S. Acute bacterial meningitis in adults. A 20-year overview. Arch Intern Med. 1997;157:425-30. http://dx.doi.org/ 10.1001/archinte.1997.00440250077009

3. Kishaba T, Uchihara T, Ueno K, Goeku C, Shimabukuro Y. Gram-negative rod meningitis probably caused by "occult" disseminated strongyloidiasis [in Japanese]. Okinawa Medical Journal. 1985;22:539-41.

4. Kishaba T, Suginohara K, Tamaki K, Miyara Y, Endo K, Taira Y, et al. Culture negative suppurative meningitis probably caused by occult disseminated strongyloidiasis [in Japanese]. Okinawa Medical Journal. 1989;26:219-21.

5. Sasaki Y, Taniguchi T, Kinjo M, McGill RL, McGill AT, Tsuha S, et al. Meningitis associated with strongyloidiasis in an area endemic for strongyloidiasis and human T-lymphotropic virus-1: a singlecenter experience in Japan between 1990 and 2010. Infection. 2013;41:1189-93. http://dx.doi.org/10.1007/s15010-013-0483-2

6. Tanaka T, Hirata T, Parrott G, Higashiarakawa M, Kinjo T, Kinjo T, et al. Relationship among Strongyloides stercoralis infection, human T-cell lymphotropic virus type 1 infection, and cancer: a 24-year cohort inpatient study in Okinawa, Japan. Am J Trop Med Hyg. 2016;94:365-70. http://dx.doi.org/10.4269/ ajtmh.15-0556
7. Cresci GA, Bawden E. Gut microbiome: what we do and don't know. Nutr Clin Pract. 2015;30:734-46. http://dx.doi.org/ $10.1177 / 0884533615609899$

8. Spanos A, Harrell FE Jr, Durack DT. Differential diagnosis of acute meningitis. An analysis of the predictive value of initial observations. JAMA. 1989;262:2700-7. http://dx.doi.org/10.1001/ jama.1989.03430190084036

9. Durand ML, Calderwood SB, Weber DJ, Miller SI, Southwick FS, Caviness VS Jr, et al. Acute bacterial meningitis in adults. A review of 493 episodes. N Engl J Med. 1993;328:21-8. http://dx.doi.org/ 10.1056/NEJM199301073280104

10. Siddiqui AA, Berk SL, Siddiqui A, Berk S. Diagnosis of Strongyloides stercoralis infection. Clin Infect Dis. 2001;33:1040 7. http://dx.doi.org/10.1086/322707

Address for correspondence: Mitsuru Mukaigawara, Department of Medicine, Okinawa Chubu Hospital, 281 Miyazato, Uruma, 9042293, Okinawa, Japan; email: mmukaigawara@gmail.com

\section{Use of Next-Generation Sequencing for Diagnosis of West Nile Virus Infection in Patient Returning to Belgium from Hungary}

\author{
Elke Wollants, ${ }^{1}$ David Smolders, ${ }^{1}$ \\ Reinout Naesens, Peggy Bruynseels, \\ Katrien Lagrou, Jelle Matthijnssens, \\ Marc Van Ranst
}

Author affiliations: : KU Leuven Rega Institute, Leuven, Belgium (E. Wollants, J. Matthijnssens, M. Van Ranst); ZNA Hospital Middelheim, Antwerp, Belgium (D. Smolders, R. Naesens, P. Bruynseels); University Hospital Leuven, Belgium (K. Lagrou, M. Van Ranst)

DOI: https://doi.org/10.3201/eid2412.180494

An elderly patient in Belgium who became critically ill after returning from Hungary was tested for pathogens using routine diagnostic tests. All results were negative. However, using next-generation sequencing on a cultured respiratory sample, laboratorians detected a complete West Nile virus genome, similar to strains isolated in southeastern Europe.

\footnotetext{
${ }^{1}$ These authors contributed equally to this article.
} 